\title{
Long Non-Coding RNA CYP4B1-PS1-001 Inhibits Proliferation and Fibrosis in Diabetic Nephropathy by Interacting with Nucleolin
}

\author{
Suyu Wang ${ }^{\mathrm{a}}$ Xin Chen ${ }^{\mathrm{a}} \quad$ Min Wang $^{\mathrm{a}} \quad$ Di Yao $^{\mathrm{a}} \quad$ Tianyu Chen $^{\mathrm{a}} \quad$ Qin Yan ${ }^{\mathrm{b}}$ \\ Weiping $\mathrm{Lu}^{\mathrm{a}}$ \\ aDepartment of Endocrinology and Metabolism, Huai'an First People's Hospital, Nanjing Medical \\ University, Jiangsu, 'Department of Microbiology, Nanjing Medical University, Nanjing, China
}

\section{Key Words}

Diabetic nephropathy $\bullet$ LncRNA $・$ Nucleolin $・$ Proliferation $・$ Fibrosis $・$ Ubiquitination

\begin{abstract}
Background/Aims: Our previous studies demonstrated that a novel long non-coding RNA, CYP4B1-PS1-001, was significantly downregulated in early diabetic nephropathy in vivo and in vitro, and CYP4B1-PS1-001 overexpression could inhibit the proliferation and fibrosis of mouse mesangial cells (MMCs). However, the underlying mechanism of the CYP4B1-PS1-001-mediated regulation of proliferation and fibrosis in diabetic nephropathy remains undetermined. Methods: RNA-protein pull-down assay, RNA-binding protein immunoprecipitation, and mass spectrometry were used to investigate CYP4B1-PS1-001 interacted with the upregulated protein nucleolin (NCL). siRNA method was applied to knockdown NCL in MMCs, the interaction between CYP4B1-PS1-001 and NCL were determined by Western blot analysis and RT-qPCR. The effect of CYP4B1-PS1-001 in the regulation of NCL was detected by cycloheximide (CHX) and ubiquitination assays. Results: We found that CYP4B1-PS1-001 interacts with NCL, and CYP4B1-PS1-001 inhibits the proliferation and fibrosis of MMCs depending on interaction with NCL. Furthermore, degradation of CYP4B1-PS1-001-associated NCL was mediated by a ubiquitin proteasome-dependent pathway. Conclusion: Our study provides evidence that CYP4B1-PS1-001 regulates the ubiquitination and degradation of NCL and thereby plays a critical role in the proliferation and fibrosis of MMCs, indicating that CYP4B1-PS1-001 and NCL may be promising prognostic biomarkers and molecular targets for the treatment of diabetic nephropathy.
\end{abstract}

S. Wang, X. Chen and M. Wang contributed equally to this work.

Qin Yan

and Weiping Lu 


\section{Introduction}

Diabetic nephropathy is a common clinical complication of diabetes that is associated with cardiovascular disease and premature mortality, and it represents the leading cause of end-stage renal disease [1]. Albuminuria is used to stage diabetic nephropathy and is regarded as a biomarker for diagnosis $[2,3]$. The histopathological characteristics of diabetic nephropathy include glomerular hypertrophy, thickening of the glomerular basement membrane, and mesangial matrix expansion, and generally the development of glomerular sclerosis and fibrosis [4]. It is believed that oxidative stress, inflammation, activated hexose, and the polyol pathway are crucially linked with the progress of diabetic nephropathy [2], which has provided scientific researchers with an enormous number of potential targets.

Long non-coding RNAs (lncRNAs) are a group of RNAs of greater than 200 nucleotides in length without protein-coding capacity [5,6], which are presumed to regulate the expression of genes by interacting with proteins, RNAs, and DNA. LncRNAs are regarded as functional and critical participants in many physiological processes, including cell proliferation, apoptosis, chromatin regulation, and cell-cycle progression, while the aberrant expression of IncRNAs and changes in their structure are associated with a wide spectrum of diseases including autoimmune and metabolic diseases, neurological and muscular disorders, and different types of cancer.

Noticeably, emerging concepts of the IncRNA-mediated control of the pathogenesis of diabetes and its complications as well as some of the challenges and therapeutic opportunities have been widely accepted [7]. Our previous study showed that a novel IncRNA, CYP4B1PS1-001, participated in early diabetic nephropathy, which was firstly selected using the lncRNA microarrays of kidney tissues from 3 obese type 2 diabetic $\mathrm{db} / \mathrm{db}$ mice, and its downregulated expression was further confirmed in vivo and in vitro. Moreover, CYP4B1PS1-001 overexpression inhibited the proliferation and fibrosis of mesangial cells, which are considered as prominent features during the early stage of diabetic nephropathy, indicating a crucial role for CYP4B1-PS1-001 in its pathogenesis [8]. However, the mechanism underlying the CYP4B1-PS1-001-mediated regulation of proliferation and fibrosis in diabetic nephropathy is unclear.

Since virtually all functional RNA molecules interact with proteins complexes, and proteins have been confirmed as the first and principal partners of lncRNAs [9], identification of CYP4B1-PS1-001-bound proteomes will gain insight into molecular mechanisms of its function in diabetic nephropathy progress. In the present study, nucleolin (NCL), served as a marker protein to monitor changes in nucleolar organization [10] and participated in the development of type 2 diabetic cardiomyopathy, was initially identified to interact with CYP4B1-PS1-001 by RNA-Protein pull-down assay, RNA-binding protein immunoprecipitation (RIP) and mass spectroscopy (MS). The decreased expression level of NCL protein and transcript was further proved in mouse mesangial cells (MMCs) and renal cortex tissues from $\mathrm{db} / \mathrm{db}$ mice, and silencing of NCL significantly inhibited proliferation and fibrosis of mesangial cells. Importantly, lentivirus-CYP4B1-PS1-001 dramatically suppressed the levels of NCL protein but not mRNA, while overexpression of NCL recruited CYP4B1PS1-001-inhibited cell proliferation and fibrosis. Mechanistic studies revealed that CYP4B1PS1-001 promoted the ubiquitination and degradation of NCL via E3 ubiquitin ligase TRIM2 and thereby the interaction between CYP4B1-PS1-001 and NCL may be promising molecular targets for the therapy of diabetic nephropathy. 


\section{Cellular Physiology Cell Physiol Biochem 2018;49:2174-2187

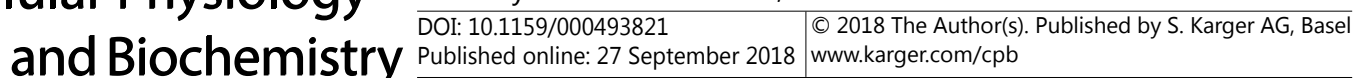 \\ Wang et al.: LncRNA/NCL in Proliferation and Fibrosis of DN}

\section{Materials and Methods}

\section{Cell Culture and Animals}

MMCs and HEK293T cells were cultured in Dulbecco's modified eagle medium (DMEM) medium with $5 \mathrm{mmol} / \mathrm{l}$ glucose and $5 \%$ fetal bovine serum (FBS) at $37^{\circ} \mathrm{C}$ in a humidified, $5 \% \mathrm{CO}_{2}$ atmosphere. All cells were obtained from Shanghai Institutes for Biological Sciences (Shanghai, China).

Characteristics and maintaining conditions of six 9-week-old male C57BL/KsJ background db/db mice and six heterozygote $\mathrm{db} / \mathrm{m}$ mice were previously described [8], while their characteristics were shown in Table 1. Renal cortical tissues dissected from kidney of $\mathrm{db} / \mathrm{db}$ and $\mathrm{db} / \mathrm{m}$ mice were collected for Western blot and quantitative real-time PCR (qPCR). The protocol was approved by the Animal Care and Ethical Committee of Nanjing Medical

University. All procedures were in accordance with the guidelines of the Institute for Laboratory Animal Research of Nanjing Medical University.

\section{Lentivirus}

Mouse NCL cDNA from MMCs was cloned in pCDH-CMV-MCS-EF1-RFP (briefly named as pCDHRFP) with three repetitive sequence of Flag tag at the C-terminus to generate the recombinant pCDHNCL transferring plasmid. To obtain the recombinant lentivirus, using Lipofectamine ${ }^{\mathrm{TM}} 2000$ transfection reagent (Invitrogen, Carlsbad, CA, USA) according to the manufacturer's instructions, the virus-packaging cells HEK293T were seeded for $24 \mathrm{~h}$ and then co-transfected with lentiviral plasmids (pCDH-NCL or pCDHRFP), packaging vector psPAX2 and envelope vector pMD2.G. The supernatants were collected $48 \mathrm{~h}$ after transfection to obtain lentivirus expressing NCL and the vector control pCDH-RFP, respectively, as well as lentvirus expressing CYP4B1-PS1-001 and the vector control pCDH-CMV-MCS-EF1-copGFP (briefly named as pCDH-GFP) in previous studies [8].

\section{RNA Interference}

Two different siRNAs against NCL (si-NCL 1\# and si-NCL 2\#) and siTRIM2 (si-TRIM2 1\# and si-TRIM2 2\#) were designed and synthesized by Ribobio (Guangzhou, China) to avoid off-target effects, as well as the negative control (si-NC). Cells were seeded at 6-well plates for $24 \mathrm{~h}$, transfected with indicated siRNA $(100 \mathrm{nmol} / \mathrm{L})$ by Lipofectamine ${ }^{\mathrm{TM}} 2000$ in serum-free medium, and harvested for following analyses. The sequences of siRNAs were listed in Table 2.

Table 1. Characteristics of $\mathrm{db} / \mathrm{m}$ and $\mathrm{db} / \mathrm{db}$ mice

\begin{tabular}{|c|c|c|c|c|c|c|c|}
\hline Case & Sample & Sex & $\begin{array}{c}\text { Age } \\
\text { (week) }\end{array}$ & $\begin{array}{l}\text { Blood glucose } \\
\text { (mmol/l) }\end{array}$ & $\begin{array}{c}\text { Body weight } \\
\text { (g) }\end{array}$ & $\begin{array}{l}\text { Urine volume } \\
\text { (ml) }\end{array}$ & $\begin{array}{l}24 \mathrm{~h} \text { urine albumin excretion } \\
\text { (UAE; } \mu \mathrm{g} / 24 \mathrm{~h} \text { ) }\end{array}$ \\
\hline \multirow{2}{*}{1} & $\mathrm{db} / \mathrm{m}-1$ & male & 9 & 10.6 & 22.9 & 0.5 & 0.217 \\
\hline & $\mathrm{db} / \mathrm{db}-1$ & male & 9 & $\geq 33.3$ & 38.1 & 14 & 3.219 \\
\hline \multirow[b]{2}{*}{2} & $\mathrm{db} / \mathrm{m}-2$ & male & 9 & 10.1 & 23.2 & 0.6 & 0.162 \\
\hline & $\mathrm{db} / \mathrm{db}-2$ & male & 9 & $\geq 33.3$ & 38.4 & 16 & 3.275 \\
\hline \multirow[b]{2}{*}{3} & $\mathrm{db} / \mathrm{m}-3$ & male & 9 & 12.0 & 22.7 & 0.5 & 0.311 \\
\hline & $\mathrm{db} / \mathrm{db}-3$ & male & 9 & $\geq 33.3$ & 36.8 & 11 & 2.462 \\
\hline \multirow{2}{*}{4} & $\mathrm{db} / \mathrm{m}-3$ & male & 9 & 10.5 & 20.2 & 0.6 & 0.407 \\
\hline & $\mathrm{db} / \mathrm{db}-3$ & male & 9 & $\geq 33.3$ & 34.8 & 15.5 & 2.869 \\
\hline \multirow{2}{*}{5} & $\mathrm{db} / \mathrm{m}-3$ & male & 9 & 11.0 & 22.1 & 0.7 & 0.314 \\
\hline & $\mathrm{db} / \mathrm{db}-3$ & male & 9 & $\geq 33.3$ & 37.0 & 13.5 & 2.912 \\
\hline \multirow[b]{2}{*}{6} & $\mathrm{db} / \mathrm{m}-3$ & male & 9 & 10.4 & 20.7 & 0.6 & 0.289 \\
\hline & $\mathrm{db} / \mathrm{db}-3$ & male & 9 & $\geq 33.3$ & 38.9 & 12.5 & 2.618 \\
\hline
\end{tabular}




\section{Cellular Physiology Cell Physiol Biochem 2018;49:2174-2187

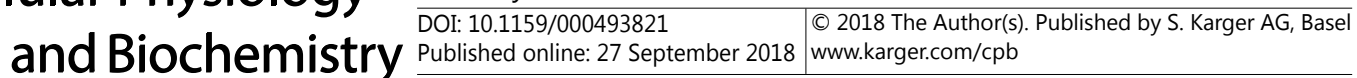 \\ Wang et al.: LncRNA/NCL in Proliferation and Fibrosis of DN}

RNA Isolation and Quantitative Real-time PCR (qPCR)

Briefly, total RNAs isolated from MMCs or mice renal cortical tissues were extracted using TRIzol reagent (Invitrogen, Carlsbad, CA, USA) following the manufacturer's instructions and subjected to the PrimeScript ${ }^{\circledR}$ RT reagent kit (TaKaRa) to obtain cDNA. qPCR was performed by the standard SYBR-Green PCR kit protocol and LightCycler480 II Sequence Detection System (Roche, Basel, Switzerland). The relative expression of the RNAs normalized to the signal generated from $\beta$-actin was calculated using the comparative cycle threshold (CT) method. The sequences of primers used in qPCR were presented in Table 2 . All of the reactions were run in triplicate, and every reaction contained three technical replicates.

\section{Western blot Analysis and Antibodies}

The procedure of Western blot was previously described [8]. Anti-proliferating cell nuclear antigen (PCNA) antibody, anti-Cyclin D1 antibody, anti-Collagen I antibody, anti-fibronectin antibody, anti- $\alpha$-tubulin antibody were described as previously [18]. Antinucleolin (1: 1 000) and anti-Flag (1: 1 000) were obtained from Abcam (Cambridge, MA, USA) and Cell Signaling Technology (Beverly, MA, USA), respectively. Species-specific horseradish peroxidase (HRP)conjugated secondary antibodies used in Western blot were purchased from Santa Cruz Biotechnology Inc (1: 4 000). Densitometry analysis was used to determine the differences of protein expression using Scion Image software (Scion Corporation, Frederick, MD, USA). Every Western blot was biological repeats at least in triplicate if not mentioned.

\section{Cell Proliferation Assay}

$3 \times 10^{3}$ cells per well was seeded in 96 -well plates at day 0 , and measured for cell proliferation by Cell Count Kit-8 (CCK-8) (Dojindo Molecular Technologies, Tokyo, Japan) according to the manufacturer's instructions.

\section{RNA-Protein Pull-down Assay}

Biotin-labeled RNAs were in vitro transcribed using Biotin RNA Labeling Mix (Roche Diagnostics, Indianapolis, IN, USA) and T7 RNA polymerase (Roche), treated with RNase-free DNase I (Roche) and purified with RNeasy Mini Kit (Thermo, CA, USA). Cell lysate (1 mg) extracted by RIPA buffer (Sigma-Aldrich, Hong Kong, China) was mixed with biotinylated RNA (50 pmol). $60 \mu$ l washed streptavidin magnetic beads (Invitrogen, Carlsbad, CA, USA) were added to each binding reaction and incubated at room temperature for $1 \mathrm{~h}$. The beads were washed briefly five times and boiled in sodium dodecyl sulfate (SDS) buffer, and the retrieved proteins were resolved by SDS-polyacrylamide gel electrophoresis (SDS-PAGE) and visualized by silver staining or Western blot. Specific bands were excised and identified by mass spectrometry (MS; Shanghai Applied Protein Technology Co. Ltd, Shanghai, China).
Table 2. The sequences of primers in qPCR assay and siRNAs in RNA interference

\begin{tabular}{|c|c|}
\hline Target & Primer \\
\hline \multirow[t]{2}{*}{ PCNA } & Forward, 5' - GGGAGCTTGGCAATGGGAACATTA -3 \\
\hline & Reverse, 5'- TGTAGGAGACAGTGGAGTGGCTTT -3' \\
\hline \multirow[t]{2}{*}{ Cyclin D1 } & Forward, 5'- CTGGCCATGAACTACCTGGA - 3 ' \\
\hline & Reverse, 5' - ATCCGCCTCTGGCATTTTGG -3' \\
\hline \multirow[t]{2}{*}{ Collagen I } & Forward, 5'- AAGGGTCCCTCTGGAGAACC -3' \\
\hline & Reverse, 5'- TCTAGAGCCAGGGAGACCCA -3' \\
\hline \multirow[t]{2}{*}{ Fibronectin } & Forward, 5'- AGACCATACCTGCCGAATGTAG -3' \\
\hline & Reverse, 5' - GAGAGCTTCCTGTCCTGTAGAG -3' \\
\hline \multirow[t]{2}{*}{$\beta$-actin } & Forward, 5'- GTGACGTTGACATCCGTAAAGA -3' \\
\hline & Reverse, 5' - GCCGGACTCATCGTACTCC -3' \\
\hline \multirow[t]{2}{*}{ NCL } & Forward, 5'- TGGAAATTGAGGGCAGAACAATC -3' \\
\hline & Reverse, 5'- TCCTCAGACAGACCTTTGACAAACA -3 \\
\hline si-NCL 1\# & AGGAAAGAAGACGAAGTTT \\
\hline si-NCL 2\# & GAAAGAAGACGAAGTTTGA \\
\hline si-TRIM2 1\# & GCCUGGAACGGUACAAGAATT \\
\hline si-TRIM2 2\# & GCTCTAAAGGGTTAGCATA \\
\hline si-NC & AATTCTCCGAACGTGTCACGT \\
\hline
\end{tabular}




\section{Cellular Physiology Cell Physiol Biochem 2018;49:2174-2187

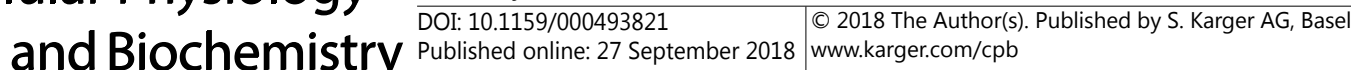 \\ Wang et al.: LncRNA/NCL in Proliferation and Fibrosis of DN}

RNA-Binding Protein Immunoprecipitation (RIP)

RIP assay was performed using Magna RIPTM RNA-Binding Protein Immunoprecipitation Kit (Millipore, Billerica, MA, USA). MMCs transduced with lentivirus-CYP4B1-PS1-001 were lysed by RIP lysis buffer. Whole cell lysate was incubated with anti-NCL antibody (Abcam, Cambridge, MA, USA) or rabbit IgG (Millipore, Billerica, MA, USA) for $3 \mathrm{~h}$ at $4^{\circ} \mathrm{C}$. Protein $\mathrm{A} / \mathrm{G}$ agarose beads were added to recruit RNA-protein complexes. RNAs associated with NCL were recovered and subjected to reverse transcription PCR (RT-PCR).

Immunoprecipitation and Ubiquitination Assay

MMCs transfected with lentivirus-CYP4B1-PS1-001 or the control were cultured in 6-well plates for 48 $\mathrm{h}$ and incubated with proteasome inhibitor MG132 (10 $\mu \mathrm{M})$ for $24 \mathrm{~h}$. After washing with ice-cold PBS and lysing in $1 \times$ RIPA buffer, total cell lysates were incubated with anti-NCL antibody overnight at $4^{\circ} \mathrm{C}$. Protein A/G-conjugated beads $(40 \mu \mathrm{l})$ were incubated for $3 \mathrm{~h}$ and washed three times with RIPA lysis buffer. Bound proteins were eluted with sample buffer and visualized by SDS-PAGE. Co-immunoprecipitated NCL was detected with anti-NCL antibody, while ubiquitinated NCL with anti-Ubiquitin antibody (CST, USA).

NCL-RFP and pCDH-GFP plasmids were transfected into MMCs. $48 \mathrm{~h}$ after transfection, the lysates were immunoprecipitated with the indicated antibodies on protein A/G beads (Life Technologies) overnight at $4{ }^{\circ} \mathrm{C}$ with rotation and then boiled in SDS buffer. The eluted proteins were detected by mass spectrometry and Western blotting.

\section{Statistical Analysis}

The SPSS 18.0 software (SPSS, Chicago, IL, USA) was used for the statistical analysis of experimental data, which presented as mean \pm standard (SD). Significance between groups was tested using paired Student's t test, Wilcoxon test or Pearson's chi-squared test. $P<0.05$ was considered to be statistically significant.

\section{Results}

CYP4B1-PS1-001 interacts with NCL

It has been shown that many lncRNAs conduct their regulatory functions in the form of RNA-protein complexes, such as chromatin-modifying complexes, transcription factors, and ribonucleoprotein complexes. To investigate the underlying mechanisms of the action of CYP4B1-PS1-001, previously indicated as a downregulated lncRNA that regulates proliferation and fibrosis in diabetic nephropathy, an RNA-protein pull-down assay was performed to identify proteins associated with CYP4B1-PS1-001 in MMCs (Fig. 1A), which were further resolved by SDS-PAGE and subjected to MS; the specifically bound proteins are listed in Table 3. MS analysis of the protein band specific to CYP4B1-PS1-001 revealed that NCL was the main associated protein, which was detected simultaneously by western blot analysis of 3 independent RNA-protein pull-down assays. To verify this result further, RIP with an antibody against NCL was also performed using cell extracts from MMCs. RTPCR analysis of the immunoprecipitates revealed that NCL was associated with endogenous CYP4B1-PS1-001. A significant enrichment of CYP4B1-PS1-001 with the anti-NCL antibody was observed. These results suggest that CYP4B1-PS1-001 may physically bind with NCL.

\section{NCL participates in proliferation and fibrosis of mesangial cells}

To investigate the role of CYP4B1-PS1-001-associated NCL in proliferation and fibrosis during the development of diabetic nephropathy, NCL expression was evaluated in MMCs. Western blot and qRT-PCR analyses showed that the expression levels of NCL protein and mRNA were significantly enhanced in MMCs under high glucose conditions compared with low glucose conditions, which respectively mimic diabetic pathological and normal physiological environments (Fig. 2A and 2B). Importantly, consistent results were simultaneously observed in 6 pairs of renal cortex tissues from $\mathrm{db} / \mathrm{db}$ and $\mathrm{db} / \mathrm{m}$ mice (Fig. 2C and 2D). Furthermore, 2 chemically synthesized siRNAs against NCL were successfully designed. As expected, knockdown of NCL by si-NCL 1\# and si-NCL 2\# resulted in a drastic 


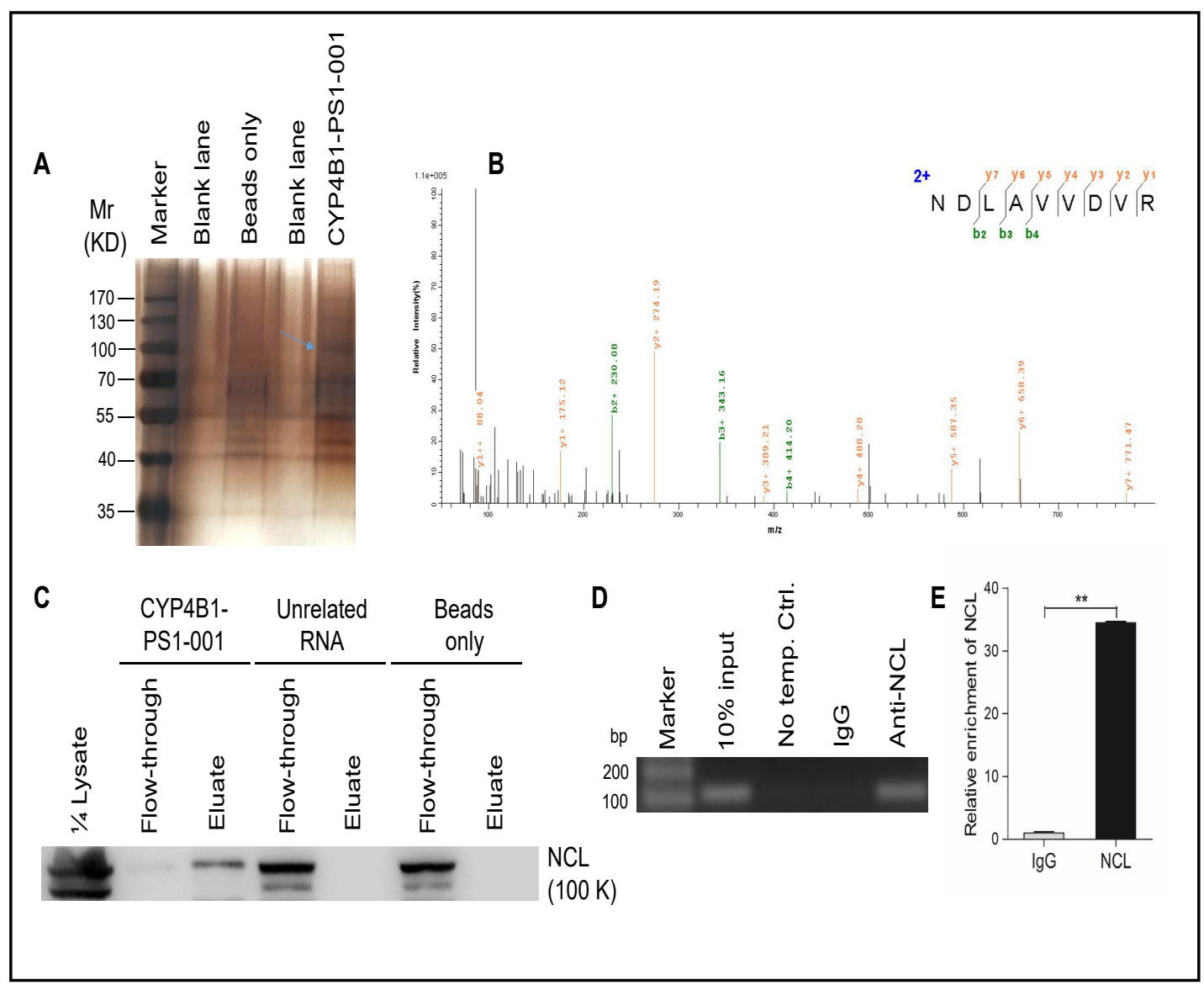

Fig. 1. Association between CYP4B1-PS1-001 and NCL in mouse mesangial cells. (A-C). RNA-Protein pulldown assay was performed for proteins immunoprecipitated from cell lysates of MMCs, and analysed by silver staining (A), mass spectrometric (B) and Western blot (C). Beads Only: proteins binding to streptavidin magnetic beads as a positive control; CYP4B1-PS1-001: proteins binding to streptavidin magnetic beads with labeled CYP4B1-PS1-001; Unrelated RNA: proteins binding to streptavidin magnetic beads with unlabeled RNA as a negative control; Mr: sizes for the proteins in the standard molecular mass ladder are given in KD. (D). RIP assay was performed using MMCs lysate incubated with anti-NCL (Anti-NCL) or rabbit IgG (IgG) as the immunoprecipitating antibodies, respectively. Purified RNA was then analyzed by RT-PCR using RIP primers specific for CYP4B1-PS1-001. All RT-PCRs were RNase sensitive (+ RNase) confirming RNA and not DNA was being assayed. 10\% Input: RT-PCR products from 10\% input lysate RNA; No temp. Ctrl.: nontemplate control. (E). Relative enrichment of endogenous NCL in RIP assay shown as (D) compared with IgG measured by RT-qPCR.

decrease in the levels of NCL protein and mRNA in MMCs under high glucose conditions. (Fig. $2 \mathrm{E}$ and $2 \mathrm{~F}$ ).

After assessing NCL silencing by synthesized siRNAs, a CCK8 assay was then employed to show the decreased growth rate of MMCs transfected with siRNAs against NCL at 2 days post-transfection (Fig. 3A). As shown in Fig. 3B and 3C, knockdown of NCL by si-NCL 1\# and si-NCL 2\# in MMCs under high glucose conditions inhibited the expression levels of PCNA and cyclin-D1 proteins, while mRNA levels observed by qRT-PCR supported these findings. The above data suggest that NCL is upregulated in MMCs and promotes proliferation during the development of diabetic nephropathy.

Since diabetic nephropathy is characterized by mesangial expansion and the accumulation of extracellular matrix (ECM), the major components of fibrosis, fibronectin and collagen I, were detected in MMCs under high glucose conditions that were transfected with siRNAs against NCL. Western blot analysis showed that knockdown of NCL decreased 
the levels of fibronectin and collagen I (Fig. 3B), while supporting results from qRT-PCR are shown in Fig. 3C.

To exclude the influence of osmolality and the increased glucose concentration on above results, the similar experiments were also performed under low glucose condition. As show in Fig. 3D and 3E, knockdown of NCL by si-NCL $1 \#$ and si-NCL $2 \#$ resulted in a drastic decreased levels of NCL protein in MMCs under low glucose to consistently inhibit the mRNA levels of PCNA, Cyclin D1, fibronectin and Collagen I. These data collectively indicate that CYP4B1-PS1-001associated NCL participates in the proliferation and fibrosis of mesangial cells.

\section{CYP4B1-PS1-001 inhibits the proliferation and fibrosis of MMCs depending on} interaction with NCL

Our previous studies indicated a potential role for CYP4B1-PS1-001 in the development of diabetic nephropathy, as well as its binding protein NCL. To determine whether the association between CYP4B1-PS1-001 and NCL is required for the proliferation and fibrosis of mesangial cells, MMCs under high glucose
Table 3. Top Abundant Proteins Interacted with CYP4B1-PS1-001 Identified by Mass Spectrometry

\begin{tabular}{|c|c|c|}
\hline Gene Name & $\begin{array}{l}\text { Protein } \\
\text { Mass }\end{array}$ & Unique Pep Count \\
\hline Nucleolin & 76722.15 & 3 \\
\hline Hnrnpab & 30830.9 & 3 \\
\hline Itih1 & 101065.7 & 2 \\
\hline Hnrnpf & 45729.37 & 1 \\
\hline Mme & 14451.46 & 1 \\
\hline Dhx9 & 149617.3 & 1 \\
\hline Mvk & 14677.92 & 1 \\
\hline A530016L24Rik & 18262.95 & 1 \\
\hline Nrac & 18347 & 1 \\
\hline Veph1 & 94500.99 & 1 \\
\hline
\end{tabular}

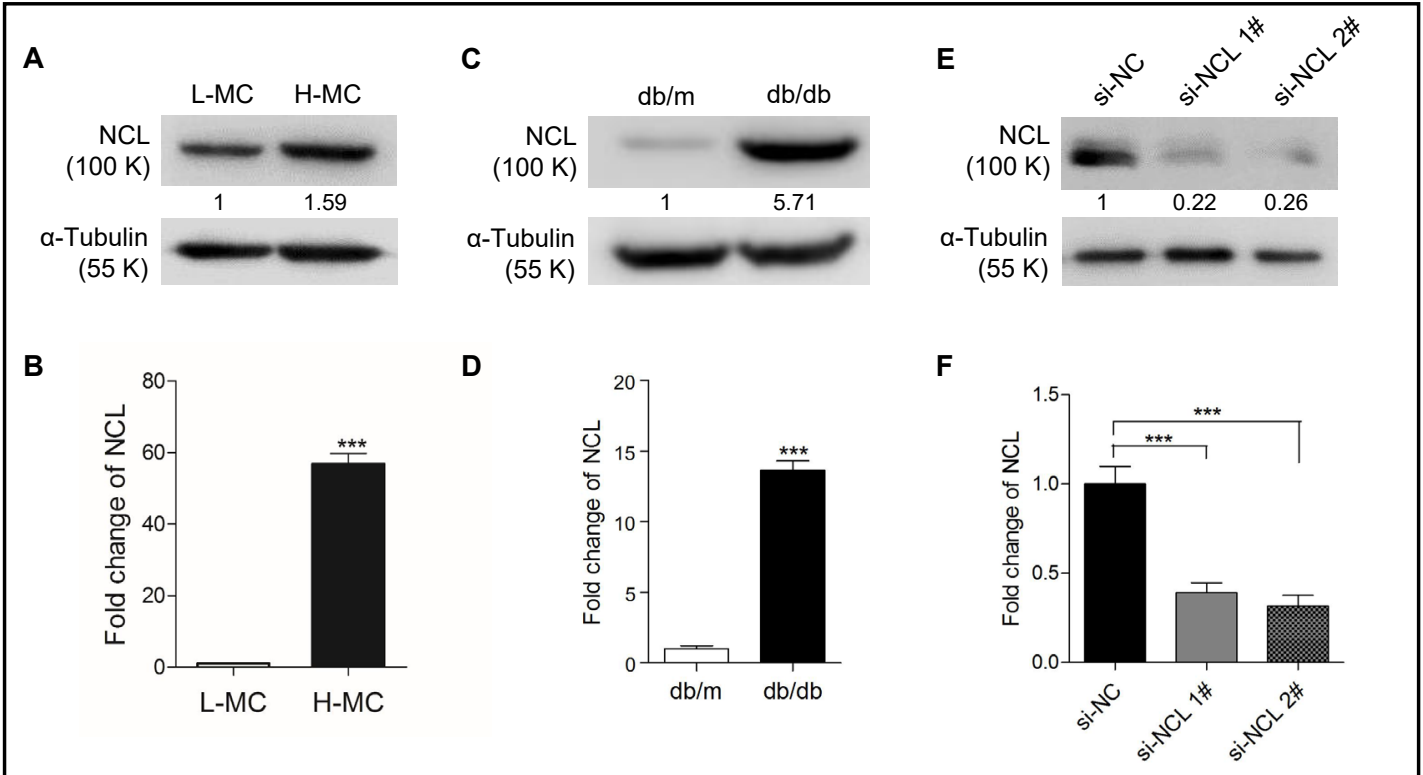

Fig. 2. Expression of NCL in cell and animal models of diabetic nephropathy and silencing of NCL. (A-B). The expression of NCL in MMCs under low-glucose (L-MC, $5 \mathrm{mM}$ ) and high-glucose (H-MC, $25 \mathrm{mM}$ ) conditions was detected by Western blot (A) and qPCR (B). The values of density of protein bands after normalization to housekeeping were shown; same for all of the following Western blot figures. ${ }^{* * *} \mathrm{P}<0.001$ for Student's t-test. (C-D). The expression of NCL in renal tissues from $\mathrm{db} / \mathrm{m}$ and $\mathrm{db} / \mathrm{db}$ mice was detected by Western blot (C) and qPCR (D). ${ }^{* * *} \mathrm{P}<0.001$ for Student's t-test. (E-F). The expression of NCL in MMCs transfected with siRNAs against NCL (si-NCL $1 \#$ and si-NCL 2\#) or the negative control (si-NC) under high-glucose condition was detected by Western blot (E) and qPCR (F). ${ }^{* * *} \mathrm{P}<0.001$ for Student's t-test. 


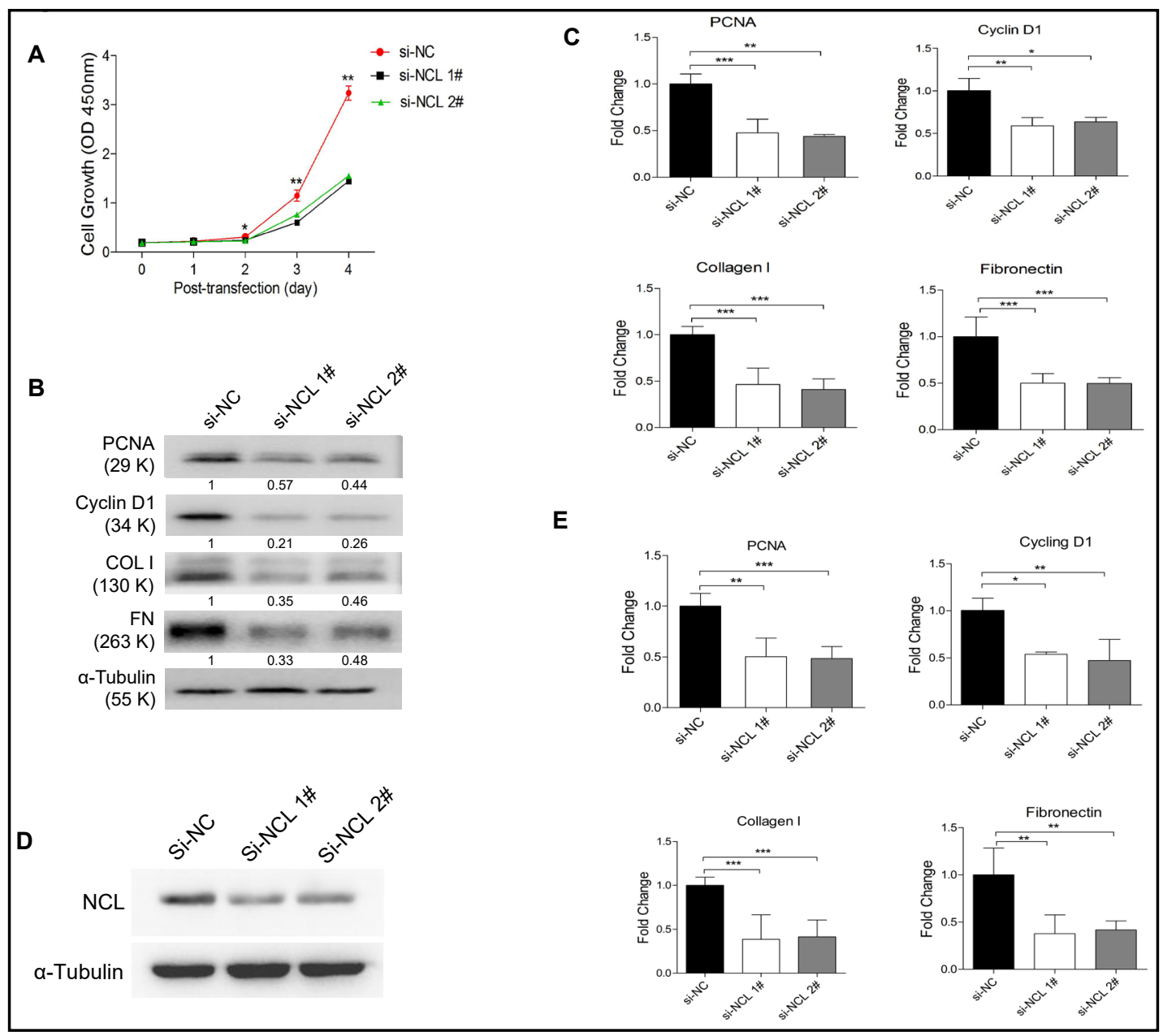

Fig. 3. Effect of NCL knockdown on proliferation and fibrosis of MMCs. (A). CCK8 assays for proliferation of MMCs transfected with siRNAs against NCL (si-NCL 1\# and si-NCL 2\#) or the negative control (si-NC) under high-glucose condition for $0,1,2,3,4$ days. ${ }^{*} \mathrm{P}<0.05$ and ${ }^{* *} \mathrm{P}<0.01$ for Student's t-test. (B-C). The expression of PCNA (PCNA), Cyclin D1 (Cyclin D1), Collagen I (COL I) and fibronectin (FN) in MMCs transfected with siRNAs against NCL (si-NCL 1\# and si-NCL 2\#) or the negative control (si-NC) under high-glucose condition for $24 \mathrm{~h}$ were detected by Western blot (B) and qPCR (C). The expression of NCL in MMCs transfected with siRNAs against NCL (si-NCL 1\# and si-NCL 2\#) or the negative control (si-NC) under low-glucose condition was detected by Western blot (D), The expression of PCNA (PCNA), Cyclin D1 (Cyclin D1), Collagen I (COL I) and fibronectin (FN) in MMCs transfected with siRNAs against NCL (si-NCL 1\# and si-NCL 2\#) or the negative control (si-NC) under low-glucose condition for $24 \mathrm{~h}$ were detected by $\mathrm{qPCR}(\mathrm{E}),{ }^{*} \mathrm{P}<0.05,{ }^{* *}$ $\mathrm{P}<0.01$ and ${ }^{* * *} \mathrm{P}<0.001$ for Student's t-test.

conditions were transduced with lentivirus expressing CYP4B1-PS1-001 or its control pCDH-GFP, which were simultaneously infected with lentivirus-NCL or its control pCDH-RFP, respectively. The expression of green fluorescent protein (GFP) and red fluorescent protein (RFP) indicated the successful infection of CYP4B1-PS1-001 or its control pCDH-GFP, and NCL or its control pCDH-RFP, respectively (Fig. 4A). As expected, qRT-PCR confirmed that the expression of NCL and CYP4B1-PS1-001 were significantly increased in MMCs transduced with lentivirus-NCL and lentivirus-CYP4B1-PS1-001, respectively (Fig. 4B).

MMCs co-transfected with lentivirus-CYP4B1-PS1-001 and lentivirus-NCL were then subjected to a CCK8 assay and western blot analysis. As a result, the decreased growth rate of MMCs with CYP4B1-PS1-001 transfection was significantly reversed by NCL overexpression (Fig. 4C). Furthermore, when CYP4B1-PS1-001 impeded the proliferation and fibrosis of

\section{KARGER}


A

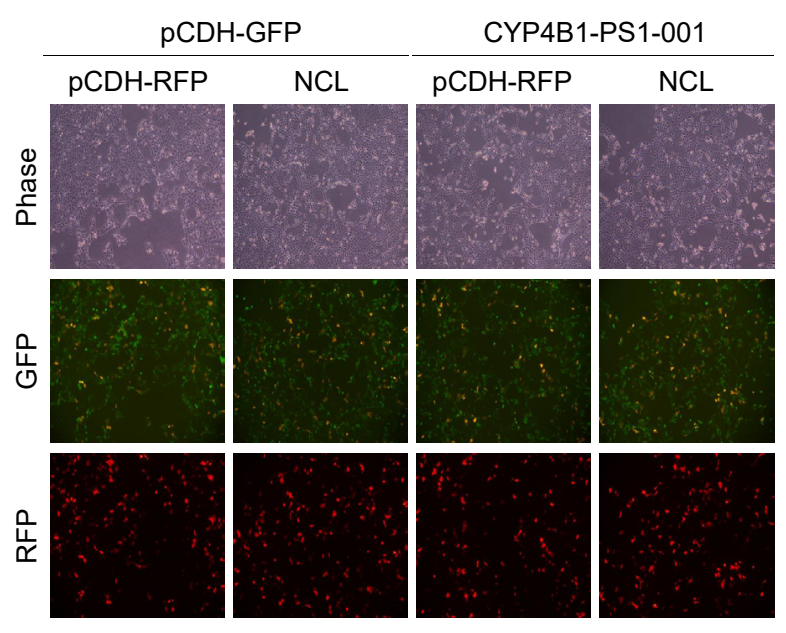

B

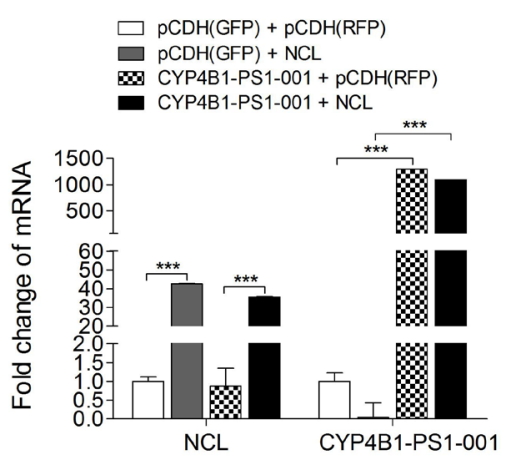

C
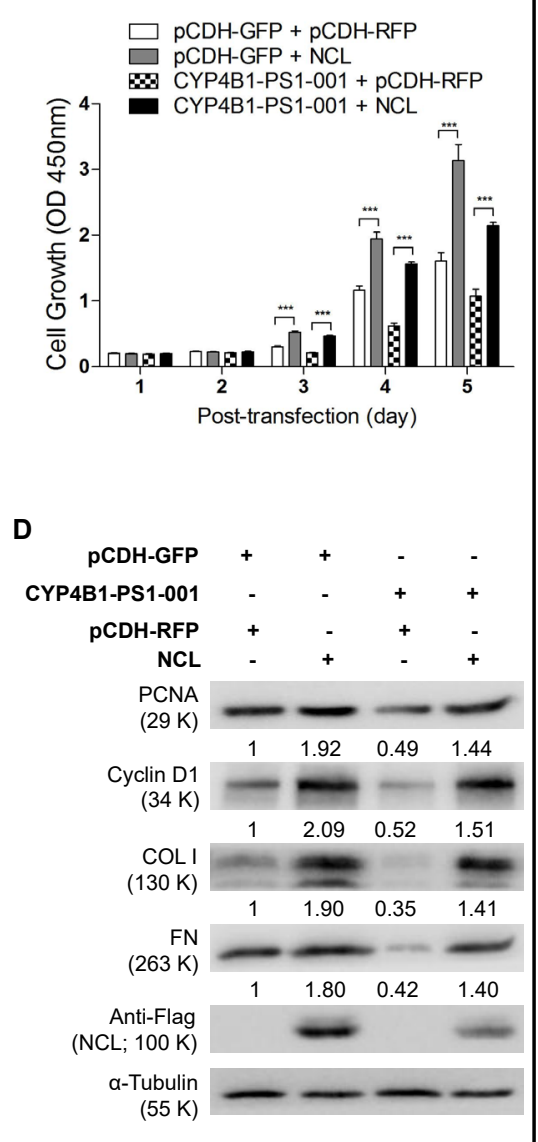

Fig.4. Recruitment of proliferation and fibrosis in CYP4B1-PS1-001-expressing MMCs by NCL overexpression. (A). MMCs were transduced with lentivirus-CYP4B1-PS1-001 (CYP4B1-PS1-001) or its control (pCDH-GFP) and co-infected with lentivirus-NCL (NCL) or its control (pCDH-RFP), and representative images were taken under light microscope (Phase, top) and fluorescent microscope (GFP, middle; RFP, bottom; original magnification, $\times 100$ ). (B). The expression levels of NCL (left lane) and CYP4B1-PS1-001 (right lane) in MMCs treated as in (A) was detected by qRT-PCR. ${ }^{*} \mathrm{P}<0.05$ and ${ }^{* * *} \mathrm{P}<0.001$ for Student's t-test. (C). CCK8 assays for proliferation of MMCs transduced with lentivirus-CYP4B1-PS1-001 or its control (pCDH-GFP) and coinfected with lentivirus-NCL or its control (pCDH-RFP) for $0,1,2,3,4,5$ days post-infection. ${ }^{* * *} \mathrm{P}<0.001$ for Student's t-test. (D). The expression levels of PCNA (PCNA), Cyclin D1 (Cyclin D1), Collagen I (COL I) and fibronectin (FN) in MMCs treated as in (A) were detected by Western blot. The antibody against Flag-tag was used to detect the exogenous expression of NCL.

MMCs as expected, lentivirus-mediated NCL expression increased the levels of PCNA and cyclin-D1 as indicators of proliferation, as well as fibronectin and collagen I as indicators of fibrosis (Fig. 4D). Collectively, these data suggest that CYP4B1-PS1-001 inhibits the proliferation and fibrosis of MMCs by interacting with NCL.

\section{CYP4B1-PS1-001 promotes ubiquitination and degradation of NCL}

It is now widely accepted that IncRNAs have regulatory roles in gene expression at both the transcriptional and post-transcriptional levels in diverse cellular contexts and biological processes. To determine whether CYP4B1-PS1-001 is involved in the regulation of NCL expression in MMCs, qRT-PCR and western blot analyses were performed to reveal that CYP4B1-PS1-001 overexpression repressed the levels of NCL protein, but not its mRNA (Fig. 5A and 5B).

\section{KARGER}




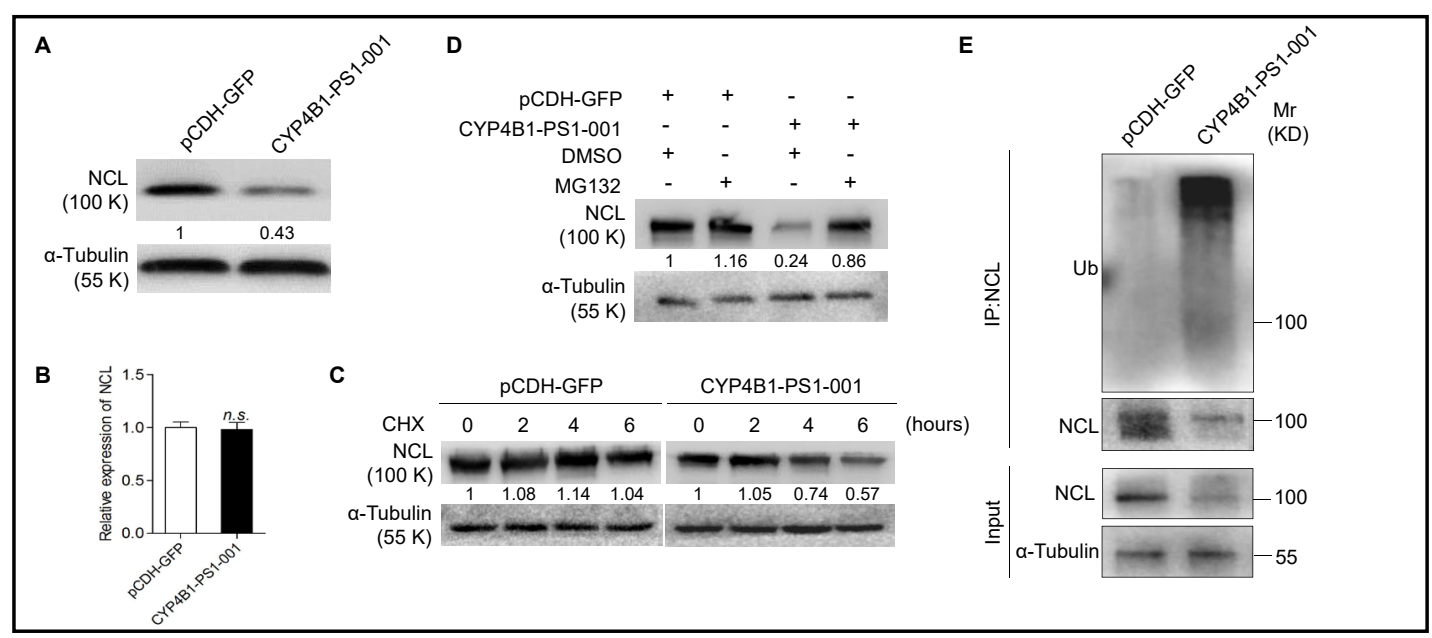

Fig. 5. Role of ubiquitination and degradation of NCL in the association with CYP4B1-PS1-001. (A-B). The expression of NCL in MMCs transduced with lentivirus-CYP4B1-PS1-001 (CYP4B1-PS1-001) or its control (pCDH-GFP) was detected by Western blot (A) and qPCR (B). n.s., not significant. (C). MMCs transducted with lentivirus-CYP4B1-PS1-001 (CYP4B1-PS1-001) or its control (pCDH-GFP) were treated with cycloheximide (CHX) for the indicated times and then subjected to Western blot with anti-NCL antibody. (D). MMCs transducted with lentivirus-CYP4B1-PS1-001 (CYP4B1-PS1-001) or its control (pCDH-GFP) were cultured in the presence (MG132) or absence (DMSO) of MG132 and then subjected to Western blot with anti-NCL antibody. (E). Lysates from MMCs that had been transduced with lentivirus-CYP4B1-PS1-001 (CYP4B1PS1-001) or its control (pCDH-GFP) and treated with MG132 were subjected to immunoprecipitation with anti-NCL antibody followed by immunoblotting analysis with anti-ubiquitin (Ub) or anti-NCL (NCL) antibodies.

Since the degradation of specific proteins by the ubiquitin proteasome-dependent pathway is a component of many cellular regulatory processes $[11 ; 12]$, we further investigated the mechanisms of CYP4B1-PS1-001-mediated NCL expression in MMCs infected with lentivirus-CYP4B1-PS1-001 or control for $24 \mathrm{~h}$ and treated with the antibacterial protein synthesis inhibitor cycloheximide (CHX) for 0, 2, 4, and 6 h. As shown in Fig. 5C, the overexpression of CYP4B1-PS1-001 reduced the stability of NCL protein in CHX-treated MMCs in a time-dependent manner, indicating the association between CYP4B1-PS1-001 and the half-life of NCL. Moreover, MMCs transduced with lentivirus-CYP4B1-PS1-001 were treated with the membrane-permeable proteasome inhibitor MG132 for $12 \mathrm{~h}$. Western blot analysis showed that treatment with MG132 inhibited the decreased level of NCL protein caused by CYP4B1-PS1-001 overexpression (Fig. 5D). Furthermore, CYP4B1-PS1-001 overexpression resulted in the obvious ubiquitination of NCL (Fig. 5E). Taken together, these results imply that CYP4B1-PS1-001 interferes with NCL protein stabilization by proteasomemediated ubiquitination and degradation.

\section{Ubiquitin Ligase TRIM2 interacts with NCL}

To investigate the underlying mechanisms of the action of NCL, the immunoprecipitation assay was performed to identify proteins associated with NCL in MMCs, which were further resolved by SDS-PAGE and subjected to MS, and the specifically binding proteins were listed in Table 4. Compared with the prediction from website STRING, MS analysis of the protein band specific to NCL revealed that E3 ubiquitin ligase TRIM2 was one of the mainly associated proteins, which were subsequently detected by immunoprecipitation assays (Fig. 6A). Then, two chemically synthesized siRNAs against TRIM2 were successfully designed. As expect, knockdown of TRIM2 by si-TRIM2 1\# and si-TRIM2 2\# resulted in a drastic decrease in the levels of TRIM2 protein and mRNA in MMCs under high glucose (Fig. 6B and 6C). Furthermore, silencing of TRIM2 elevated the levels of NCL protein (Fig. 6D), implying the role of TRIM2 as a E3 ubiquitin ligase in NCL degradation. To explore the relationship 
between CYP4B1-PS1-001 and TRIM2 in NCL degradation, RIP with RT-PCR analysis was performed to reveal that TRIM2 was not directly associated with CYP4B1-PS1-001 (Fig. 6E), suggesting that TRIM2 may physically bind with NCL, but not associated with CYP4B1-PS1-001.

\section{Discussion}

The dysregulation of IncRNAs expression and function is increasingly being implicated in the renal response to hyperglycemia and the progression of diabetic nephropathy, and related therapeutic strategies are gradually being explored $[13,14]$. Several IncRNAs have been identified and described in diabetic nephropathy process, such as PVT1 (plasmacytoma variant translocation 1), which was the first lncRNA associated with kidney disease and shown to play roles in the increased accumulation of ECM in the glomeruli, a characteristic of diabetic nephropathy $[15,16]$. Moreover, PVT1-derived microRNA-1207-5p is expressed abundantly in kidney cells and is upregulated by glucose and TGF- $\beta 1$ [17]. In our previous study, we demonstrated that 2 downregulated IncRNAs screened by lncRNA microarray analysis were associated with diabetic nephropathy, namely,
Table 4. Top Abundant Proteins Interacted with NCL identified by Mass Spectrometry

\begin{tabular}{|c|c|c|}
\hline Gene Name & Protein Mass & Unique Pep Count \\
\hline Pkm & 57844.26 & 8 \\
\hline Eef1a1 & 50113.26 & 7 \\
\hline Actc1 & 42117.66 & 7 \\
\hline Hspa8 & 60801.17 & 7 \\
\hline Eno1 & 47140.27 & 6 \\
\hline Rplp0 & 34216.02 & 6 \\
\hline Hist1h2bf & 13936 & 6 \\
\hline Cltc & 191983.8 & 6 \\
\hline Rpl12 & 17804.35 & 5 \\
\hline Hspa1b & 70175.43 & 5 \\
\hline Phb & 29819.71 & 4 \\
\hline Hist1h1c & 21266.37 & 4 \\
\hline Npm1 & 32559.71 & 3 \\
\hline Actr1a & 42613.22 & 3 \\
\hline Trim2 & 84336.42 & 1 \\
\hline Acly & 120793.8 & 1 \\
\hline
\end{tabular}

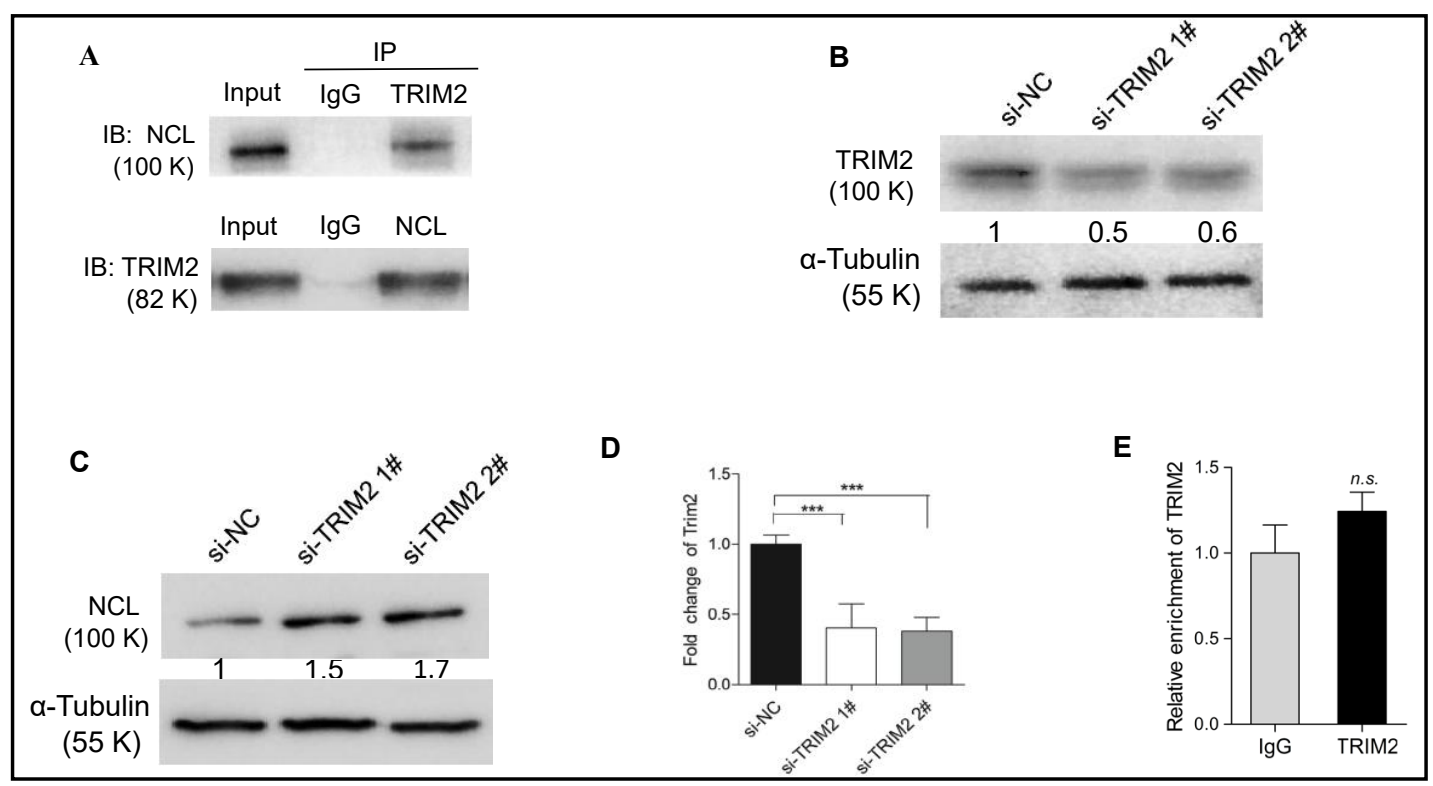

Fig. 6. Association between NCL and TRIM2 in mouse mesangial cells. (A). TRIM2 was immunoprecipitated with an anti-TRIM2 antibody and co-immunoprecipitated NCL was detected by immunoblotting with an anti-NCL antibody (above lane), while NCL was immunoprecipitated with an anti-NCL antibody and coimmunoprecipitated TRIM2 was detected by immunoblotting with an anti-TRIM2 antibody (below lane). (B-C). The expression of TRIM2 in MMCs transfected with siRNAs against TRIM2 (si-TRIM2 1\# and siTRIM2 2\#) or the negative control (si-NC) under high-glucose condition was detected by Western blot (B) and RT-qPCR (C). ${ }^{* * *} \mathrm{P}<0.001$ for Student's t-test. (D). The expression of NCL in MMCs transduced with siTRIM2 (si-TRIM2 1\# and si-TRIM2 2\#) or the negative control (si-NC) was detected by Western blot. (E). Relative enrichment of endogenous TRIM2 in RIP assay compared with IgG measured by RT-qPCR. n.s., not significant. 
CYP4B1-PS1-001 and ENSMUST00000147869, and the overexpression of both of these lncRNAs inhibited the proliferation and fibrosis of mesangial cells [18]. These earlier studies encouraged us to explore the molecular mechanism underlying the lncRNA-mediated pathogenesis of diabetic nephropathy.

Increasing evidences has indicated that lncRNAs play important roles in regulating gene expression at epigenetic, transcriptional and post-transcriptional levels via complicated mechanisms, including splicing regulation and translational control [19], competing with endogenous mRNAs for miRNA binding [20-22], eliciting transcriptional silencing by providing binding sites for small RNAs [23], and altering protein activity [24]. According to the pervasiveness of protein-RNA interactions, many reports underline the various functions that lncRNAs serve, for example as a "guide" to recruit protein complexes to target genes, as a "scaffold" to assemble proteins into RNPs, and as a "decoy" to sequester regulatory proteins away from target genes [25]. Here, we demonstrated a new strategy in that CYP4B1-PS1-001 facilitates the ubiquitination and degradation of NCL and thereby plays a critical role in the proliferation and fibrosis of MMCs.

NCL is a multifunctional protein localized primarily in the nucleolus and involved in several aspects of DNA metabolic and RNA regulatory mechanisms, such as transcription, ribosome assembly, mRNA stability and translation, and microRNA processing [26]; therefore, NCL is closely associated with the regulation of cell proliferation and growth. Correspondingly, NCL dysregulation presented as a sequence-specific RNA-binding protein that is closely linked with the pathogenesis of a number of diseases, such as viral infection, autoimmune diseases, Alzheimer's disease, Parkinson's disease, and cancer development. As for the role of NCL in diabetes and its complications, the high levels of myocardial NCL have been explored in rat models of diabetic cardiomyopathy [27]; moreover, due to its response to pharmacological agents, NCL has been identified as a target that is especially sensitive to pharmacological AMPK activators and thus represents a potential biomarker for the development of drugs that diminish diabetic renal hypertrophy [28].

In this study, we first investigated whether NCL was involved in proliferation and fibrosis in diabetic nephropathy by interacting with CYP4B1-PS1-001. Interestingly, CYP4B1-PS1-001 overexpression resulted in a significant decrease in the level of NCL protein, while altered CYP4B1-PS1-001 expression had no influence on the level of NCL mRNA. It is well known that, in addition to autophagy-lysosomes, the ubiquitin/proteasome proteolytic system is the major pathway responsible for the highly regulated extra-lysosomal degradation of cytosolic, nuclear, and endoplasmic reticulum proteins in eukaryotic cells. Via the degradation of a broad array of proteins, the ubiquitin/proteasome system is highly regulated and responsible for the maintenance of protein homeostasis and adaptation to environmental changes. Most proteins contain at least one or more lysine specific ubiquitination sites, and the ubiquitination process is catalyzed by the sequential actions of E1 ubiquitin-activating, E2 ubiquitin-conjugating, and E3 ubiquitin ligase enzymes, followed by protein transfer to the $26 \mathrm{~S}$ proteasome. NCL is also subjected to proteasomal degradation via ubiquitination, since a herpes virus-associated ubiquitin-specific protease (HAUSP) regulates the stability of NCL by removing ubiquitin moieties [29]. In this study, CHX-chase analysis demonstrated that the half-life of NCL protein was shortened in MMCs overexpressing CYP4B1-PS1-001. Further experiments revealed that CYP4B1-PS1-001 mainly enhanced non-ubiquitinated NCL protein levels when its stability was increased by the proteasome inhibitor MG132. Subsequent immunoprecipitation experiments confirmed that CYP4B1-PS1-001 promoted NCL ubiquitination in MMCs. These results provide evidence that CYP4B1-PS1-001 interferes with NCL protein stability by enhancing its ubiquitination and degradation in MMCs. This may be one of the mechanisms through which NCL participates in the CYP4B1-PS1-001mediated anti-proliferation and anti-fibrosis response during diabetic nephropathy. Further studies will be needed to better understand the other mechanisms by which CYP4B1-PS1-001 regulates NCL and the possible involvement of other proteins, RNAs or DNAs interacted with CYP4B1-PS1-001 in this process. 
Here, we propose that TRIM2 functions as an E3 ubiquitin ligase to mediate NCL ubiquitination protein. TRIM2 is a member of the TRIM super family of E3 ligase proteins containing at least 37 members, which contains a consensus RING domain, B-Box, and coiledcoil structures in the amino terminus. The RING domain is the molecular signature of the E3 ligase. Unlike other TRIM family proteins that contain two B-box domains, TRIM2 contains only one B-box domain, although the significance of this is as yet unclear.

Even though the question of whether TRIM2 is a protein that is directly connected with NCL or controls the targeting of NCL to chromatin needs further investigation, our biochemical data strongly support a model in which TRIM2 is negatively controlled by NCL. Our results showed that TRIM2 can function as an E3 ligase to interact with NCL via the ubiquitin-proteasome degradation pathway. However, at the present time, the details of how TRIM2 physically interacts with NCL and their pathogenesis remain unclear. Our data strongly supports a negative regulation of TRIM2 in NCL stability; however, which domains of NCL are critical for the ubiquitination of TRIM2 is needed to be determined in future study. Nevertheless, since knockdown of TRIM2 moderately alleviated on NCL abundance, we cannot completely exclude the possibility that other E3 ubiquitin ligases may also involved in CYP4B1-PS1-001-mediated NCL degradation.

\section{Conclusion}

In summary, on the basis of a previous study of the lncRNA CYP4B1-PS1-001-mediated inhibition of proliferation and fibrosis during diabetic nephropathy, this work further identified the underlying mechanism of its associated protein NCL and that the CYP4B1PS1-001/NCL axis via the ubiquitin-proteasome pathway may serve as prognostic biomarkers and effective targets for the treatment of diabetic nephropathy.

\section{Acknowledgements}

We are grateful to Professor Chun Lu from Nanjing Medical University for critical reading of the manuscript. Weiping Lu received Research project of Jiangsu provincial health and Family Planning Commission, grant H201458.

\section{Disclosure Statement}

The authors declare that no conflicts of interest exist.

\section{References}

1 Groop PH, Thomas MC, Moran JL, Waden J, Thorn LM, Makinen VP, Rosengard-Barlund M, Saraheimo M, Hietala K, Heikkila O, Forsblom C, FinnDiane Study G: The presence and severity of chronic kidney disease predicts all-cause mortality in type 1 diabetes. Diabetes 2009;58:1651-1658.

-2 Arora MK, Singh UK: Molecular mechanisms in the pathogenesis of diabetic nephropathy: an update. Vascul Pharmacol 2013;58:259-271.

3 Kitada M, Kanasaki K, Koya D: Clinical therapeutic strategies for early stage of diabetic kidney disease. World J Diabetes 2014;5:342-356.

4 Risdon RA, Sloper JC, De Wardener HE: Relationship between renal function and histological changes found in renal-biopsy specimens from patients with persistent glomerular nephritis. Lancet 1968;2:363-366. 


\section{Cellular Physiology Cell Physiol Biochem 2018;49:2174-2187 and Biochemistry Published \begin{tabular}{l|l} 
DOI: 10.1159/000493821 & $\begin{array}{l}\text { (c) } 2018 \text { The Author(s). Published by S. Karger AG, Basel } \\
\text { www.karger.com/cpb }\end{array}$
\end{tabular} \\ Published online: 27 September 2018 www.karger.com/cpb}

5 Derrien T, Johnson R, Bussotti G, Tanzer A, Djebali S, Tilgner H, Guernec G, Martin D, Merkel A, Knowles DG, Lagarde J, Veeravalli L, Ruan X, Ruan Y, Lassmann T, Carninci P, Brown JB, Lipovich L, Gonzalez JM, Thomas M, Davis CA, Shiekhattar R, Gingeras TR, Hubbard TJ, Notredame C, Harrow J, Guigo R: The GENCODE v7 catalog of human long noncoding RNAs: analysis of their gene structure, evolution, and expression. Genome Res 2012;22:1775-1789.

6 Banfai B, Jia H, Khatun J, Wood E, Risk B, Gundling WE, Jr., Kundaje A, Gunawardena HP, Yu Y, Xie L, Krajewski K, Strahl BD, Chen X, Bickel P, Giddings MC, Brown JB, Lipovich L: Long noncoding RNAs are rarely translated in two human cell lines. Genome Res 2012;22:1646-1657.

-7 Najafian B, Alpers CE, Fogo AB: Pathology of human diabetic nephropathy. Contrib Nephrol 2011;170:3647.

8 Wang M, Wang S, Yao D, Yan Q, Lu W: A novel long non-coding RNA CYP4B1-PS1-001 regulates proliferation and fibrosis in diabetic nephropathy. Mol Cell Endocrinol 2016;426:136-145.

9 Chu C, Spitale RC, Chang HY: Technologies to probe functions and mechanisms of long noncoding RNAs. Nat Struct Mol Biol 2015;22:29-35.

10 Kodiha M, Banski P, Stochaj U: Computer-based fluorescence quantification: a novel approach to study nucleolar biology. BMC Cell Biol 2011;12:25.

11 Lecker SH, Goldberg AL, Mitch WE: Protein degradation by the ubiquitin-proteasome pathway in normal and disease states. J Am Soc Nephrol 2006;17:1807-1819.

12 Wang J, Maldonado MA: The ubiquitin-proteasome system and its role in inflammatory and autoimmune diseases. Cell Mol Immunol 2006;3:255-261.

13 Wapinski O, Chang HY: Long noncoding RNAs and human disease. Trends Cell Biol 2011;21:354-361.

14 Amaral PP, Mattick JS: Noncoding RNA in development. Mamm Genome 2008;19:454-492.

15 Guan Y, Kuo WL, Stilwell JL, Takano H, Lapuk AV, Fridlyand J, Mao JH, Yu M, Miller MA, Santos JL, Kalloger SE, Carlson JW, Ginzinger DG, Celniker SE, Mills GB, Huntsman DG, Gray JW: Amplification of PVT1 contributes to the pathophysiology of ovarian and breast cancer. Clin Cancer Res 2007;13:5745-5755.

16 Alvarez ML, DiStefano JK: Functional characterization of the plasmacytoma variant translocation 1 gene (PVT1) in diabetic nephropathy. PLoS One 2011;6:e18671.

17 Alvarez ML, Khosroheidari M, Eddy E, Kiefer J: Role of microRNA 1207-5P and its host gene, the long noncoding RNA Pvt1, as mediators of extracellular matrix accumulation in the kidney: implications for diabetic nephropathy. PLoS One 2013;8:e77468.

18 Wang M, Yao D, Wang S, Yan Q, Lu W: Long non-coding RNA ENSMUST00000147869 protects mesangial cells from proliferation and fibrosis induced by diabetic nephropathy. Endocrine 2016;54:81-92.

19 Guttman M, Rinn JL: Modular regulatory principles of large non-coding RNAs. Nature 2012;482:339-346.

-20 Poliseno L, Salmena L, Zhang J, Carver B, Haveman WJ, Pandolfi PP: A coding-independent function of gene and pseudogene mRNAs regulates tumour biology. Nature 2010;465:1033-1038.

21 Jeck WR, Sharpless NE: Detecting and characterizing circular RNAs. Nat Biotechnol 2014;32:453-461.

22 Wierzbicki AT, Ream TS, Haag JR, Pikaard CS: RNA polymerase V transcription guides ARGONAUTE4 to chromatin. Nat Genet 2009;41:630-634.

23 Franco-Zorrilla JM, Valli A, Todesco M, Mateos I, Puga MI, Rubio-Somoza I, Leyva A, Weigel D, Garcia JA, Paz-Ares J: Target mimicry provides a new mechanism for regulation of microRNA activity. Nat Genet 2007;39:1033-1037.

-24 Iyer MK, Niknafs YS, Malik R, Singhal U, Sahu A, Hosono Y, Barrette TR, Prensner JR, Evans JR, Zhao S, Poliakov A, Cao X, Dhanasekaran SM, Wu YM, Robinson DR, Beer DG, Feng FY, Iyer HK, Chinnaiyan AM: The landscape of long noncoding RNAs in the human transcriptome. Nat Genet 2015;47:199-208.

25 Yang Y, Wen L, Zhu H: Unveiling the hidden function of long non-coding RNA by identifying its major partner-protein. Cell Biosci 2015;5:59.

26 Abdelmohsen K, Gorospe M: RNA-binding protein nucleolin in disease. RNA Biol 2012;9:799-808.

27 Sun L, He X, Wang J, Jiang B, Tong Z, Liu Y, Li Y, Xiao X: [Nucleolus expression in diabetic cardiomyopathy]. Zhong Nan Da Xue Xue Bao Yi Xue Ban 2014;39:1056-1060.

28 Kodiha M, Salimi A, Wang YM, Stochaj U: Pharmacological AMP kinase activators target the nucleolar organization and control cell proliferation. PLoS One 2014;9:e88087.

29 Lim KH, Park JJ, Gu BH, Kim JO, Park SG, Baek KH: HAUSP-nucleolin interaction is regulated by p53-Mdm2 complex in response to DNA damage response. Sci Rep 2015;5:12793. 